\title{
Magnetic Quantum-Dot Cellular Automata: Recent Developments and Prospects
}

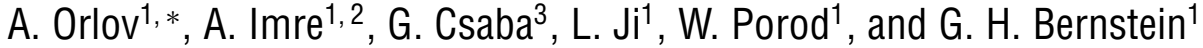 \\ ${ }^{1}$ University of Notre Dame, Center for Nano Science and Technology, Notre Dame, IN 46556, USA \\ ${ }^{2}$ Currently at Argonne National Laboratory, Materials Science Division and Center for Nanoscale Materials, \\ Argonne, IL 60439, USA \\ ${ }^{3}$ Technical University of Munich, Institute for Nanoelectronics, Munich, D-80333, Germany
}

\begin{abstract}
Quantum-dot Cellular Automata (QCA) is a computational paradigm that uses local physical coupling between nominally identical bistable building blocks (cells) assembled into arrays to perform binary logic functions. QCA offers low power dissipation and high integration density of functional elements. Depending upon the choice of local fields causing interactions between the cells, different types of QCA are possible, such as magnetic, electronic, or optical. Here we discuss recent developments in the field of magnetic QCA (MQCA) all-magnetic logic where planar, magnetically-coupled, nanometer-scale magnets are assembled into the networks that perform binary computation. The nanomagnets are defined by electron beam lithography. We demonstrate the operation of basic elements of MQCA architecture such as binary wire, three input majority logic gate, and their combination, and discuss interfacing such systems with conventional CMOS-based logic.
\end{abstract}

Keywords:

\section{CONTENTS}

1. Introduction $\ldots \ldots \ldots \ldots \ldots \ldots \ldots \ldots \ldots \ldots \ldots \ldots \ldots \ldots$

2. Fabrication, Measurements, and Simulations

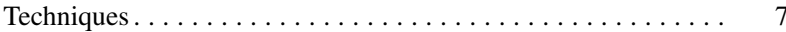

3. MQCA Devices: Binary Wires $\ldots \ldots \ldots \ldots \ldots \ldots \ldots \ldots \ldots \ldots$

4. MQCA Devices: Majority Logic Gate ................ 8

5. MLG-Binary Wire Combination Device . . . . . . . . . . 11

6. Development of the Input and Readout for MQCA . . . . . . . . 11

7. Alternative Techniques for MQCA Fabrication . . . . . . . . 12

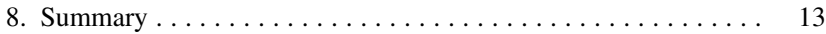
Acknowledgments ....................... 13

References and Notes ................... 13

\section{INTRODUCTION}

The use of the phenomenon of magnetism for information processing goes back to the end of XIX century. In 1888, Oberlin Smith suggested the use of permanent magnetic impressions for the recording of sound. The recording of the human voice on a steel piano wire was first carried out in 1898 by a Danish inventor Valdemar Poulsen, whose invention gave rise some 30 years later to a magnetic tape recording industry. With the creation of the first computers, the use of magnetic storage elements

*Author to whom correspondence should be addressed. such as tapes, cores, and later magnetic disks, have become widespread.

Early on in the computer era, several attempts were made to develop all-magnetic logic, most notably using such devices as "laddics" and "transfluxors." These devices were magnetic ferrite elements of complex shape, interconnected by windings of copper wire. For example, the laddic $^{1}$ was an element that had the appearance of a small ladder cut out of a ferrite with wire windings serving as inputs and outputs. By controlling the switching path through the structure, any Boolean function could be produced. The switching speeds of a few tenths of a microsecond and repetition rates of a few hundred $\mathrm{kHz}$ were reported. ${ }^{2}$ During the infancy of semiconductor processing, these numbers looked rather attractive. Moreover, even almost half a century later, all-magnetic logic devices are still unsurpassed in terms of their reliability, nonvolatile data retention and radiation hardness.

In the early sixties, several functional all-magnetic computers, which were able to withstand the electromagnetic pulses from nuclear detonations and lightning surges, were built for niche applications such as aeronautics and railroad depots. One such computer was built for the United States Air Force in $1962 .{ }^{3}$ With a clock rate of $600 \mathrm{kHz}$, it was capable of performing more than 12,000 additions or subtractions of 24-bit words per second. The processor's 
magnetic core memory could hold 400 17-bit words. With a weight of $19 \mathrm{lb}$ and a volume of $0.5 \mathrm{ft}^{3}$ it consumed only about $90 \mathrm{~W}$ of power. All logic and memory functions were implemented with magnetic elements; nonmagnetic circuitry included only the clock generator and sense amplifiers to read out the results of calculations from memory. Later, a few notable attempts to develop all-magnetic logic were made based on using domain tip propagation logic (DTPL) ${ }^{4-6}$ (late 1960's) and magnetic bubbles ${ }^{7}$ (1970's). What prevented the all-magnetic logic in the 1960's and the 1970's from developing a permanent stronghold in the computer world, alas, was the introduction of the integrated circuits and the development of CMOS technology.
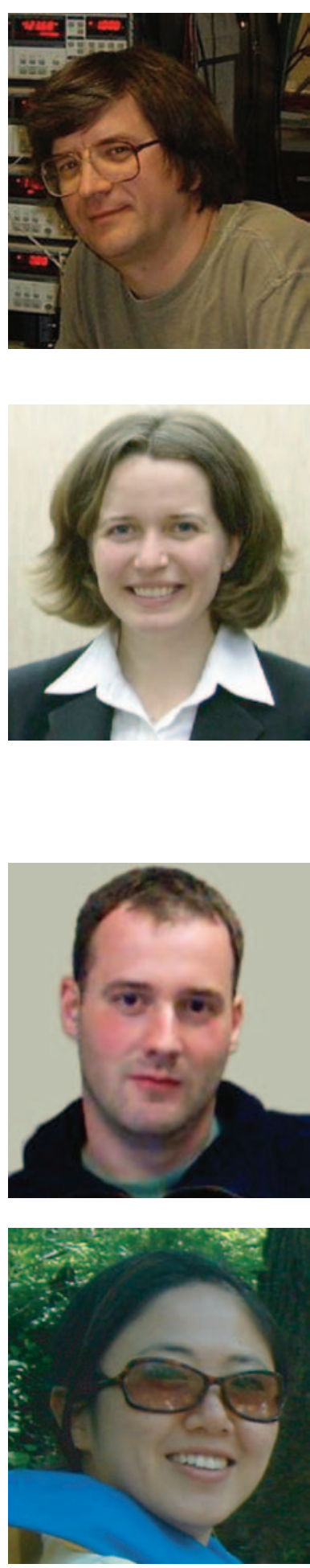

Alexei Orlov received his M.S. degree in Physics from the Moscow State University in 1983. From 1983 to 1993 he worked at the Institute of Radio Engineering and Electronics of the Russian Academy of Sciences, Moscow. During this time he conducted research on mesoscopic and quantum ballistic effects in electron transport of GaAs field-effect transistors. He received his Ph.D. from the same Institute in 1990. He was a visiting fellow at the University of Exeter, UK in 1993. He joined the Department of Electrical Engineering at the University of Notre Dame, IN, in 1994 as a Research Assistant Professor and was promoted to rank of Research Associate Professor in 2000. His topics of research include experimental studies of mesoscopic, single-electron and molecular electronic devices and sensors, nanomagnetics and quantum-dot cellular automata. Alexei Orlov has authored or co-authored more than 60 journal publications.

Alexandra Imre has received her M.S. degree in Electrical Engineering from the Budapest University of Technology and Economics, Hungary, in 2001, in the field of microelectronics and biomedical engineering; and Ph.D. degree from the University of Notre Dame, IN, in 2005 where she conducted research on dipole-coupled nanomagnets for quantum-dot cellular automata (QCA) logic applications. Her experimental work included fabrication and measurement of ferromagnetic computing systems on silicon wafer. Dr. Imre is currently a joint post-doctoral appointee at the Magnetism and Superconductivity Group of the Materials Science Division at Argonne National Laboratory, and at the Nanofabrication Group of the Center for Nanoscale Materials at Argonne National Laboratory (Illinois, US). Her work covers various micro- and nanofabrication techniques and metrologies, with special emphasis on focused ion-beam based lithography and rapid prototyping. Her present scientific research investigates the generation, propagation, and the possible applications of surface plasmon polaritons on noble metal surfaces.

György Csaba was born in Budapest, Hungary, in 1974. He received the M.S. degree from the Technical University of Budapest in 1998 and his Ph.D. degree from the University of Notre Dame in 2003. He is currently working as a research assistant at the Technical University of Munich, Germany. His research interests are in circuit-level modeling of nanoscale systems (especially magnetic devices) and exploring their applications for nonconventional architectures.

Lili Ji was born in Shanghai, China, in 1980. She received her B.S. degree from the Shanghai Jiaotong University in 2002. She is currently working toward the Ph.D. degree at the University of Notre Dame, Notre Dame, IN. Her research interests are in experimental studies and simulation of nanoscale magnetic systems with emphasis on the magnetic domain wall motion. 


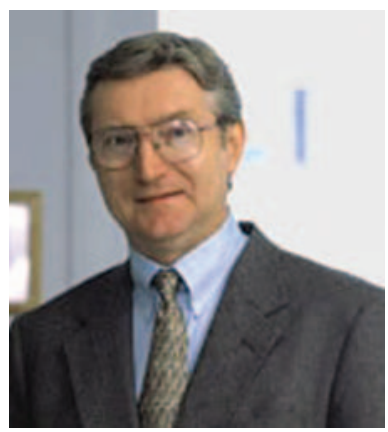

Wolfgang Porod currently is Frank M. Freimann Professor of Electrical Engineering at the University of Notre Dame. He received his Diplom (M.S.) and Ph.D. degrees from the University of Graz, Austria, in 1979 and 1981, respectively. After appointments as a postdoctoral fellow at Colorado State University and as a senior research analyst at Arizona State University, he joined the University of Notre Dame in 1986 as an Associate Professor. He now also serves as the Director of Notre Dame's Center for Nano Science and Technology. His research interests are in the area of nanoelectronics, with an emphasis on new circuit concepts for novel devices. He has authored some 300 publications and presentations. He is a Fellow of the IEEE and he has served (2002-2003) as the Vice President for Publications on the IEEE Nanotechnology Council. He also has been appointed an Associate Editor for the IEEE Transactions on Nanotechnology (2001-2005). He is a Founding Member of the IEEE Circuits and Systems Society's Technical Committee on Nanoelectronics and Gigascale Systems, and he has been active in organizing Special Sessions and Tutorials, and as a speaker in IEEE Distinguished Lecturer Programs.

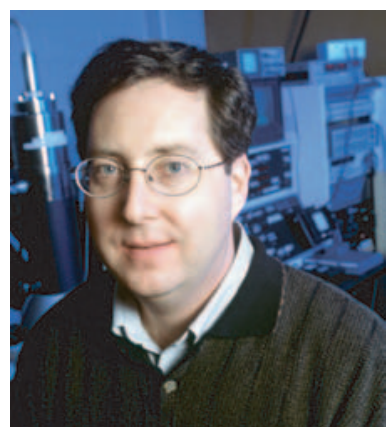

Gary H. Bernstein received the BSEE from the University of Connecticut, Storrs, with honors in 1979 and the MSEE from Purdue University, W. Lafayette, Indiana in 1981. During the summers of 1979 and '80, he was a graduate assistant at Los Alamos National Laboratory, and in the summer of 1983 interned at the Motorola Semiconductor Research and Development Laboratory, Phoenix, Arizona. He received his Ph.D. from Arizona State University, Tempe, in 1987, after which he spent a year there as a postdoctoral fellow. He joined the Department of Electrical Engineering at the University of Notre Dame, Notre Dame, Indiana, in 1988 as an assistant professor, and was the founding Director of the Notre Dame Nanoelectronics Facility from 1989 to 1998. Dr. Bernstein received an NSF White House Presidential Faculty Fellowship in 1992, was promoted to rank of Professor in 1998, and served as the Associate Chairman of his Department from 1999 to 2006. Dr. Bernstein has authored or co-authored more than 150 publications in the areas of electron beam lithography, quantum electronics, high-speed integrated circuits, electromigration, MEMS, and electronics packaging. Bernstein was named a Fellow of the IEEE in 2006.

Despite the extraordinary success in magnetic data storage over the past 50 years, ${ }^{a}$ the exploration of magnetic phenomena for logic has remained in the state of obscurity for the reasons mentioned above. However, at the beginning of the 21 st century as the semiconductor industry faces difficulties associated with the "red brick wall" for further scaling of CMOS transistors, all-magnetic logic based on nanomagnets may become an attractive alternative. The use of new fabrication methods, developments in the field of magnetic sensors, such as the discovery of the giant magnetoresistive effect and magnetic tunnel junctions, makes it possible to pursue all-magnetic logic devices on a different, submicron scale. Such devices may have advantages over CMOS in a variety of applications, as they naturally provide non-volatility, radiation hardness and high integration densities. Possible applications may include nonvolatile logic, ultra-low-power applications

\footnotetext{
${ }^{a}$ Since the time that all-magnetic logic was put aside, tremendous success was achieved in the field of magnetic storage of information. For example, the first hard disk drives introduced by IBM in 1957 had a storage density of $2000 \mathrm{bits} / \mathrm{in}^{2}$, while the storage density in the newest hard drives using perpendicular recording exceeds $200 \mathrm{Gbits} / \mathrm{in}^{2}$. We can only imagine where the technology would be if this kind of effort had gone into all-magnetic logic.
}

(which is an increasingly important trait in a world of mobile and wearable computing), computing in radiationhard environments, and architectures incorporating magnetic random access memory (MRAM). Magnetic logic devices can be very dense and continue to operate well when scaled to small sizes. In particular, unlike CMOS, they do not suffer from effects leading to intolerable power dissipation upon ultimate scaling. The downscaling of the magnets is ultimately restricted by the fact that the energy barriers separating the magnetization states have to be larger than thermal energy, $k_{\mathrm{B}} T$, and the switching speed is limited by the precession frequency. These restrictions still yield impressive integration densities of $10^{10} \mathrm{~cm}^{-2}$, and switching time on the order of nanosecond.

Several recent proposals for all-magnetic logic using metal ferromagnetic structures include MRAMbased logic, ${ }^{8}$ magnetic domain-wall logic, ${ }^{9}$ and magnetic quantum-dot cellular automata (MQCA),${ }^{10}$ which uses lithographically-defined nanomagnets. A different approach for magnetic logic involves manipulating spinpolarized electrons in a semiconductor magnetic material, where information is represented as either "spin up" or "spin down" electrons. The search for a suitable ferromagnetic semiconductor is under way, ${ }^{11}$ but no functioning device has been reported yet. 
The QCA approach is, in our opinion, the most suitable existing technology for building future all-magnetic binary logic circuits. In the generalized QCA architecture, ${ }^{12}$ binary logic is achieved with simple, nominally identical, bistable units, called "cells," that are locally connected to each other solely by field-coupling forces. The logic function performed by QCA is defined by the physical placement of the array of cells, which can be realized in different physical systems. The original proposal called for the use of electric-field-coupled arrays of semiconductor quantum dots (which gave the "quantum-dot" name to this paradigm) employing the Coulomb interactions between single electrons in the dots of the cells to perform binary operations. Several key elements of electronic QCA (EQCA) were experimentally demonstrated: a cell $;{ }^{13}$ the logic gate $;{ }^{14}$ the shift register; $;{ }^{15}$ and fanout. ${ }^{16}$ Micron-sized aluminium islands ("dots") separated by small $\left(50 \times 50 \mathrm{~nm}^{2}\right)$ oxide tunnel junctions were used in these experiments conducted at sub-Kelvin temperatures. Low-temperature conditions are required since the barrier separating the cell polarization states (that is, the energy difference between the ground and excited states, the so-called "kink" energy, $E_{\mathrm{K}}$ ) is fairly low in this EQCA embodiment, ${ }^{17} E_{\mathrm{K}} \approx 0.1 \mathrm{meV}$. If the cell size were reduced to molecular scales, room temperature EQCA operation could be attained; ${ }^{18,19}$ however, the technology for assembling molecular EQCA has not yet been developed. As discussed below, one of the significant advantages of magnetic versus electronic QCA is that it is fully functional at room temperature because of the much larger kink energies (on the order of several $\mathrm{eV}$ for submicron permalloy magnets easily attainable by modern lithography). ${ }^{20,21}$

The QCA paradigm is flexible-the same principle of local field coupling of identical cells can be extended to arrays of coupled nanomagnets. The information in this version of QCA, i.e., magnetic QCA, or MQCA, is represented by polarization of magnetic cells. The realization of MQCA-type coupling between elements was first demonstrated in Ref. [22] where the chains of $110 \mathrm{~nm}$ diameter ferromagnetic ${ }^{b}$ disks manifested ordered behavior. However, circular nanomagnets exhibit no shapeinduced anisotropy, so there is no intrinsic bistability, the existence of which is a fundamental requirement of QCA architectures. ${ }^{12}$ Bistability ensures that the cell state remains locked in the presence of external influences such as thermal fluctuations. The number of stable magnetization states in a nanomagnet is determined by its magnetic anisotropy (e.g., crystalline or shape anisotropy). We exploited the shape-induced anisotropy of elongated permalloy $\left(\mathrm{Fe}_{20} \mathrm{Ni}_{80}\right)$ nanomagnets to achieve bistability in the magnetization properties. ${ }^{20}$ Figure 1(a) shows how the hysteresis curve of elongated single-domain nanomagnets

\footnotetext{
${ }^{b}$ Supermalloy $\left(\mathrm{Ni}_{80} \mathrm{Fe}_{14} \mathrm{Mo}_{5} X_{1}\right.$, where $X$ is other metals) was used in these experiments.
}
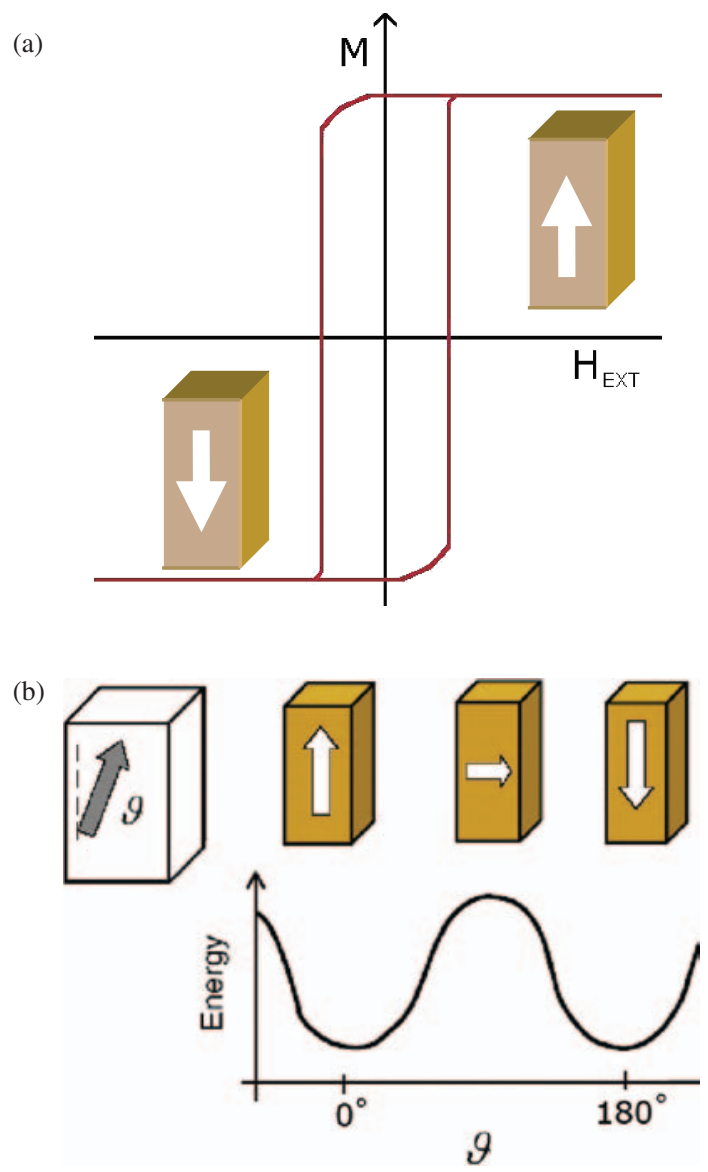

Fig. 1. (a) A hysteresis curve of an elongated nanomagnet: magnetization versus magnetic field applied parallel to the easy axis. (b) Angular dependence of remanent magnetization. Two remanent states are separated by an energy barrier. For permalloy magnets discussed in the paper this barrier is about 100 times greater than the energy of thermal fluctuations at room temperature.

results naturally in this bistability, so that the bit values ' 0 ' and ' 1 ' can be assigned to the two stable ground states. The nanomagnet's bistability arises from its remanent magnetization (magnetization at zero external magnetic field) that points along the long axis. We refer to the long axis as the "easy" axis, and the short axis as the "hard" axis. In a sufficiently strong magnetic field perpendicular to the long axis, i.e., along the hard axis, the magnetization will be forced to align with the field, but when the magnetic field is relaxed, the magnetization switches back to either of the two remanent states. The size of the magnets is chosen to be in the range of 30 to $70 \mathrm{~nm}$ on edge, and a few tens of nm thick, which is optimal since such magnets are small enough to be singledomain, but large enough to be stable against thermal fluctuations. Indeed, the energy barrier that separates two possible remanent states (Fig. 1(b)) is very high for permalloy magnets of that size, $E_{\mathrm{K}} \geq 5 \mathrm{eV}$.

Magnetic flux lines close outside of the magnets, creating strong stray fields that can be used to couple elements in close proximity through dipole-dipole 
(a)

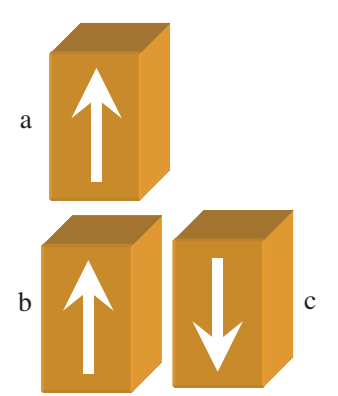

(b)

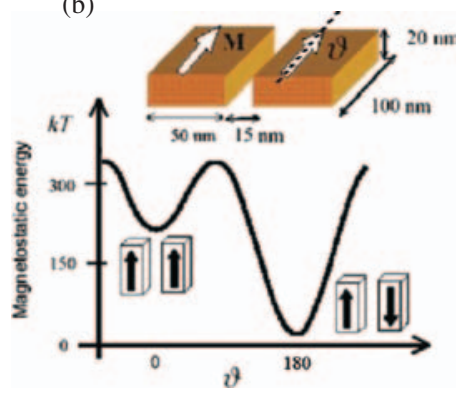

Fig. 2. (a) Two types of coupling between nanomagnets. Ferromagnetic coupling (magnets $\mathbf{a}$ and $\mathbf{b}$ ) and antiferromagnetic coupling (magnets $\mathbf{b}$ and c). All the magnets are in the ground state in the absence of an external magnetic field. (b) Energy diagram of two AF-coupled permalloy magnets in the ground and metastable states. Note that the barrier height separating two states is high enough for the magnets to stay in the metastable state at room temperature for a very long time ( $>10$ years).

interactions. When nanomagnets are placed close to each other, two types of coupling occur (Fig. 2(a)), namely, ferromagnetic (F) coupling, which occurs for magnets with their short edges adjacent and antiferromagnetic (AF) coupling, which occurs for magnets with their long edges adjacent. Due to their dipole interactions, two closely spaced, F-coupled nanomagnets prefer parallel alignment of their magnetic moments, and closely spaced AF-coupled nanomagnets prefer antiparallel alignment of their magnetic moments. Although it is energetically unfavorable for $\mathrm{F}(\mathrm{AF})$-coupled magnets to be in an antiparallel (parallel) configuration of their magnetic moments, adjacent magnets can also remain in these "wrong," metastable states (Fig. 2(b)). Due to the shape anisotropy, the magnet will remain in this state at room temperature for very long time (years), even though the metastable state has higher energy than the ground state, because the height of the barrier separating these two states (Fig. 2(b)) is on the order of the barrier separating two ground states in Figure 1(b).

A computation involving MQCA proceeds as illustrated in Figure 3. A collection of inputs at the edge of an array

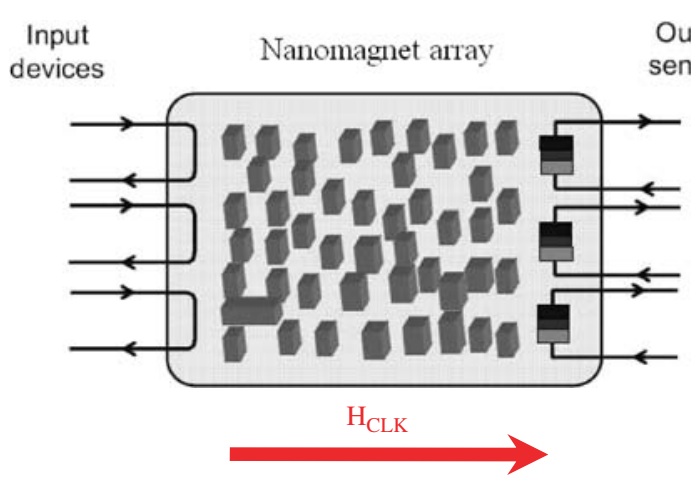

Fig. 3. Illustration of a magnetic QCA processor. The information flows from the input devices toward the output devices via magnetic interactions. Clocking magnetic field in plane of the chip $\left(H_{\mathrm{CLK}}\right)$ controls the switching process. (Here and below the direction of the magnetization is indicated by the arrow.)

of magnets influences the ground state of the array of magnets. The propagation of information from left to right results in an output state measured by sensing devices. The vast majority of the nanomagnets within the array need not be accessed externally.

What makes it possible for information to propagate over a large number of adjacent nanomagnets and result in some useful computation? The magnets must be allowed to find their ground state, but even if the inputs are strong enough to flip their adjacent magnets, the switching process will not propagate, since field coupling between magnets is not strong enough to force neighboring magnets to overcome their metastable states.

Therefore, in order to move an array of nanomagnets from an arbitrary initial state to its ground state, an external magnetic field must be used to overcome the barriers separating the states. In the case of elongated magnets, this field is parallel to the hard axis. The details of this process are illustrated in Figure 4 for a linear array of magnets. In the initial phase (Fig. 4(a)) the magnets are in some arbitrary state (a metastable state is illustrated). No external magnetic fields are yet applied. When an external magnetic field that is sufficiently strong to rotate the magnetization of the magnets is applied along the hard axis, it rotates the magnetic moments of all of the magnets horizontally into a neutral ("null") logic state (Fig. 4(b)). By the end of the first phase, the 'memory' of the structure is erased: the magnetic moments of the magnets are in line with the external field regardless of their initial state. This is an unstable state of the system, and when the field is removed, the nanomagnets relax into the AF ordered ground state, as shown in Figure 4(d). If no other magnetic fields are present, the probability for each of the two final ground states are equal $(p=0.5)$. This situation changes in the presence of a local magnetic field (created by an input device schematically shown as a current carrying wire in Fig. 4(c)) that influences only the first nanomagnet in the chain during the relaxation. The final state of the (a)

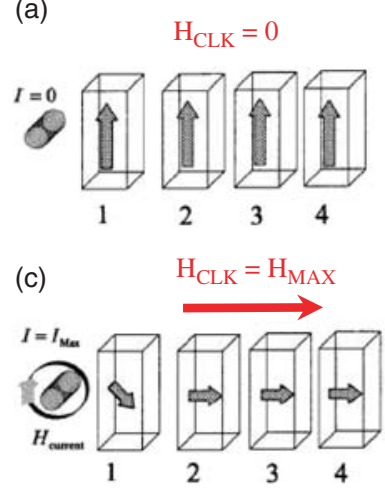

(d) $\quad \mathrm{H}_{\mathrm{CLK}}=0$

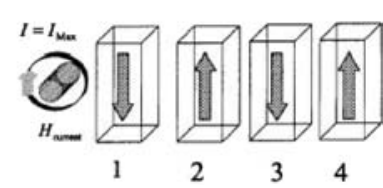

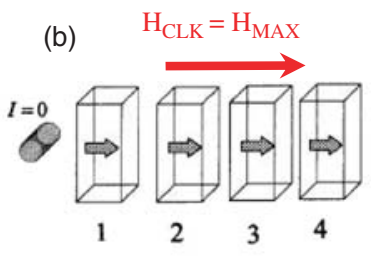

Fig. 4. Clocked control of nanomagnets switching. The initial metastable state (a) is eliminated by an external clocking field, $H_{\mathrm{CLK}}(\mathrm{b})$. By slowly releasing the clocking field (c), the system relaxes to the zerofield ground state (d). 
first magnet is thus defined by the input magnetic field. Then, due to dipole coupling, the state of the whole chain will also switch to the state determined by the input. ${ }^{c}$

The external field described above plays the combined roles of the clock and power supply in conventional digital circuits. The presence of the external clocking field (electrical for EQCA, and magnetic for MQCA) is critical to the proper operation of QCA-based systems. It was pointed out early in the development of all-magnetic logic that "the fundamental problem... is to obtain the stepby step propagation of digital information along directed paths of a network of bit storage locations without degradation of the signals." 23 In QCA this problem is solved by using a clocking field that acts as an additional source of energy (on top of the energy supplied by the input) resulting in predictable switching dynamics. Similarly to a power supply in conventional electronics, the energy supplied by a clocking field allows logic level restoration and power gain. Clocking also prevents the QCA system from being trapped in an undesirable metastable state,${ }^{24}$ can be used to realize pipelined circuits, and ensures the unidirectionality of the computation. ${ }^{10}$ For MQCA this is achieved by applying the external clocking magnetic field along the hard axis, which puts the magnets into the logic null state; a further reduction of the clocking field to zero allows the magnetic system to settle to its ground state, which in turn depends on the polarization of the input devices.

If the external clocking field is applied and removed sufficiently slowly, then the magnets always stay very close to the actual ground state. Theory ${ }^{25}$ shows that the farther the magnetization vector is from its ground state, the larger the dissipation. If the effective field ${ }^{d}$ remains almost parallel to the magnetization direction during the entire switching process, then the dissipation is minimized. Figure 5 illustrates the trade-off between clocking speed and power dissipation per nanomagnet with the dimensions shown in the figure. The minimum time necessary for nanomagnet switching is limited by magnetization precession in the nanomagnets ${ }^{26}$ and is on the order of $100 \mathrm{ps}$ for the magnets of the size shown in Figure 5. The adiabatic pumping scheme increases the clock cycle time in MQCA devices by about two orders of magnitude, but it eliminates precession in the switching process and ensures predictable operation. Our simulations show that the nanomagnets of that size will dissipate below $1 \mathrm{eV}$ per switching event. As a worst case estimate of the power dissipation in an MQCA system at clock frequency of $100 \mathrm{MHz}$, and assuming that all nanomagnets switch in each clock cycle, $10^{10}$ magnets

\footnotetext{
${ }^{c}$ Once clocking field is removed the input magnetic field plays no role in the behavior of the array and can be safely switched off.

${ }^{d}$ The effective field is the sum of the external field, the exchange interaction field, and the demagnetization field of the nanomagnet. If the clocking field is weak, the sum of exchange and demagnetization fields is dominant and will be parallel to the magnetization for the elongated nanomagnets shown in Figure 5.
}

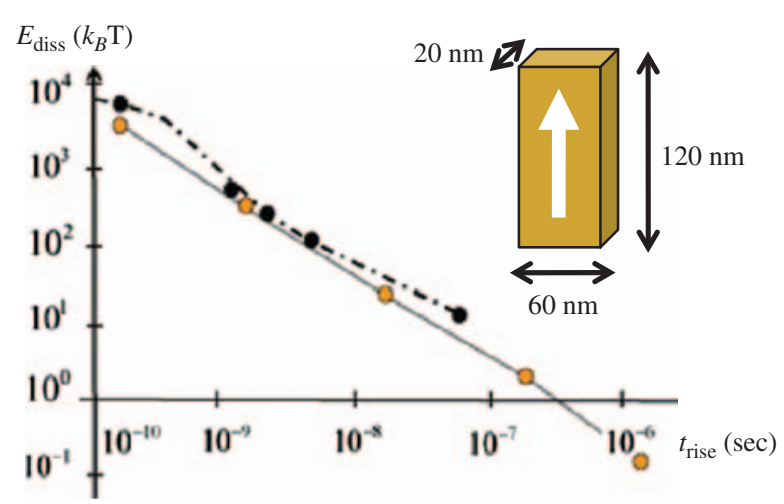

Fig. 5. Energy dissipated during the switching of a perfect single domain (solid line) and a nearly-perfect single-domain magnet (dotted line). Energy scale is calibrated in units of "room temperature $k_{\mathrm{B}} T$," i.e., $26 \mathrm{meV}$. The line are the guide for the eye.

would dissipate about a tenth of a watt. This number is somewhat misleading because the dissipation in the clock circuitry must be taken into account. Depending on the implementation of the clock circuitry, the power dissipation due to current flows in the clocking lines is about 3 to 100 times larger. Still, the simulations show that at a $100 \mathrm{MHz}$ clock speed the improvement of 2-3 orders of magnitude over CMOS based logic is possible. ${ }^{27}$

One important difference between MQCA and EQCA is in the way that the clocking field manipulates the barriers. In EQCA, the clock field is applied to raise energy barriers between the dots in the cell, to bring the cell out of null state, and to "freeze" the charge polarization of the cell in accordance with the input signal. When the clocking field is set to zero, no information is stored in an EQCA cell. For MQCA, the situation is the exact opposite; the application of the clocking field brings an MQCA cell to the null state. This difference shows one important advantage

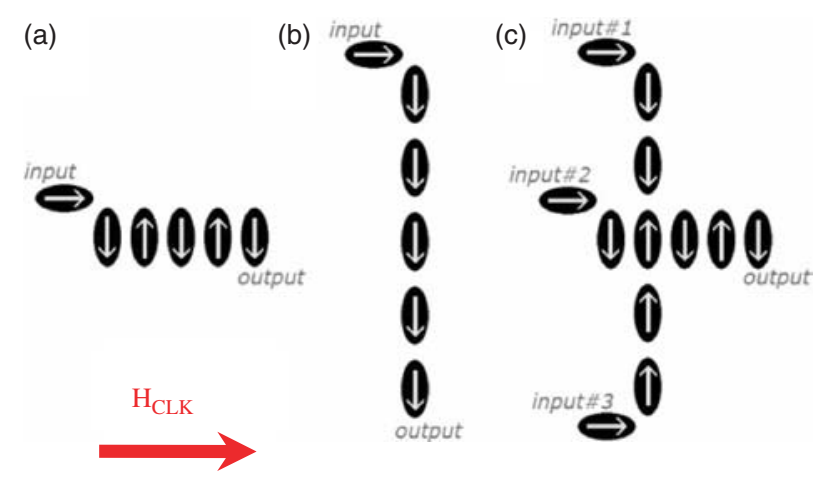

Fig. 6. Planar QCA devices built of elongated nanomagnets: (a) Antiferromagnetically-coupled binary wire, (b) ferromagneticallycoupled binary wire, and (c) a combination of a logic gate and three binary wires (two, driven by inputs \#1 and \#3, are F-coupled; one, driven by input \#2 is AF-coupled). The nanomagnet at the intersection assumes the magnetization according to the majority vote of the upper, left and lower neighbors that are driven by input magnets. The result of the majority operation is transmitted to the right. The white arrows indicate the magnetization states of the nanomagnets. Red arrow indicates the direction of the clocking field which results in such ordering. 
of MQCA over EQCA, namely the natural capacity of MQCA cells is to work as non-volatile memory elements as no power supply is required to store information.

In the original proposal ${ }^{20}$ it was suggested to use pillar-shaped nanomagnets with out-of-plane anisotropy to implement the MQCA. In this case, only AF-coupling between magnets could be employed, which has the advantage of presenting uniform coupling between nanomagnets independently of the geometric arrangement, whereas the $\mathrm{AF}$ and $\mathrm{F}$ coupling do not present the same degree of interaction. In planar MQCA, it is necessary to use both AF and $\mathrm{F}$ coupling to achieve logic gates. However, from the fabrication standpoint, particularly for the proof of concept, it is much easier to fabricate planar nanomagnets, with in-plane anisotropy.

Several examples of planar MQCA devices tested experimentally are shown in Figure 6 and will be discussed below.

\section{FABRICATION, MEASUREMENTS, AND SIMULATIONS TECHNIQUES}

The nanomagnets forming MQCA devices were fabricated by our group using electron-beam lithography (EBL), NiFe evaporation and lift-off. First, poly-methyl-methacrylate (PMMA) was spun on the surface of an oxidized silicon wafer. After evaporation, the lift-off was done in a mixture of methylene chloride and acetone at a ratio of 8:1 respectively, at room temperature. The thickness of the patterned film was in the range of 30 to $40 \mathrm{~nm}$. The magnetizing process, i.e., the application of the clocking field, was performed in the homogenous field of an electromagnet capable of a maximum $7 \mathrm{kOe}$. Due to limitations imposed by the ramping rate of the generated field, the frequency of the clocking field was below $0.01 \mathrm{~Hz}$ in our experiments.

The resulting magnetic configurations of the devices were imaged by both atomic force microscopy (AFM) and magnetic force microscopy (MFM). Magnetic force microscopy images were taken in a Digital Instruments Nanoscope IV with standard magnetic probes. Dynamics of the magnetization processes in MQCA devices were simulated by means of the freeware micromagnetic solver, OOMMF. ${ }^{28}$

\section{MQCA DEVICES: BINARY WIRES}

A linear arrangement of nanomagnets coupled only to nearest neighbors forms a "binary wire" (Figs. 6(a), (b)) where information propagates along the wire entirely via magnetic interactions. Let us consider first the lines of horizontally-aligned, vertically-(along $Y$ axis) magnetized, AF-coupled nanomagnets ${ }^{e}$ schematically shown in

\footnotetext{
${ }^{e}$ Note that the AF-coupled wire with odd number of magnets also serves as an inverter for input magnetic field signal.
}

Figure 6(a). For each binary wire, nanomagnet that is orthogonally oriented, i.e., elongated along the $X$ axis, is placed at the edge of the wire. Note that the magnet is vertically displaced from the center of adjacent magnet in the line. That allows us to use this magnet as the input device to set the state of the linear array by magnetizing it using the clocking field, with magnetization vector perpendicular to the line of magnets.

In the absence of these input magnets, the AFC line takes on one of the two possible complementary alternating dipole configurations (Fig. 7) with a 50\% probability. Setting the state of the input magnet by an external clocking field in the $X$-direction favors one of the two possible complementary states of the line. The horizontal clocking field serves two purposes: (1) it sets the state of the horizontal input magnets and (2) for sufficiently strong clocking fields (several hundred Oe for the magnets used in the experiments) forces the magnetization vectors of all nanomagnets to align horizontally (null state). However, this state will persist only so long as the clocking field is maintained. Once the clocking field is reduced to zero, the nanomagnets will return to their ground state with vertical magnetization along the easy axis. The crucial point here is that the switching behavior from the null state to one of the vertical magnetizations is strongly influenced by any additional fields, emanated by either the AF-coupled neighbors or the input magnet. While without the input magnet the probability of reaching either of vertical magnetizations is equal, this symmetry is broken in its presence. The results of micromagnetic simulations are shown in Figure 8 for an AF-coupled binary wire composed of 16 nanomagnets. ${ }^{29}$ Initially, the magnitude of the clocking field is $1500 \mathrm{Oe}$, and the wire is in the null state. Once the magnetic field is reduced to $1000 \mathrm{Oe}$, the time evolution begins (from Figs. 8(a)-(f)).

Simulations ${ }^{21}$ show that it becomes energetically favorable for the first magnet in the wire to switch into that state which provides flux closure for magnetic field lines

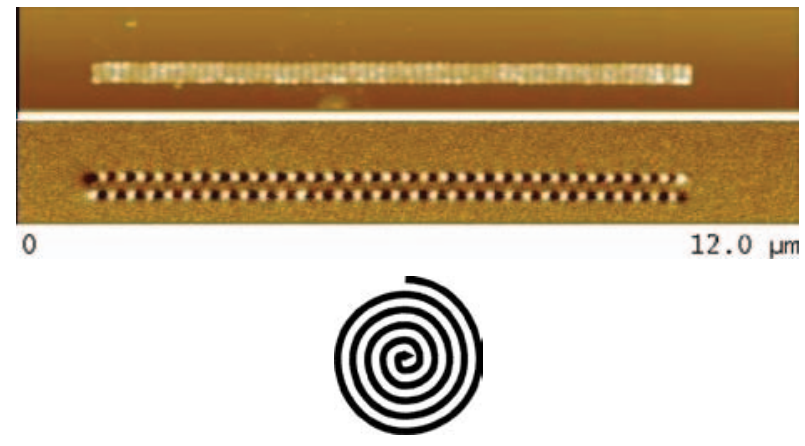

Fig. 7. An example of AF-coupled binary wire composed of 64 nanomagnets showing perfect ordering behavior. Top - AFM image, bottom - MFM image. The image is taken after a demagnetizing procedure where a rotating clocking field is slowly reduced (rotating and reducing field is indicated by a spiral). The resulting magnetization of the whole wire is therefore in one of the two possible ground states. 


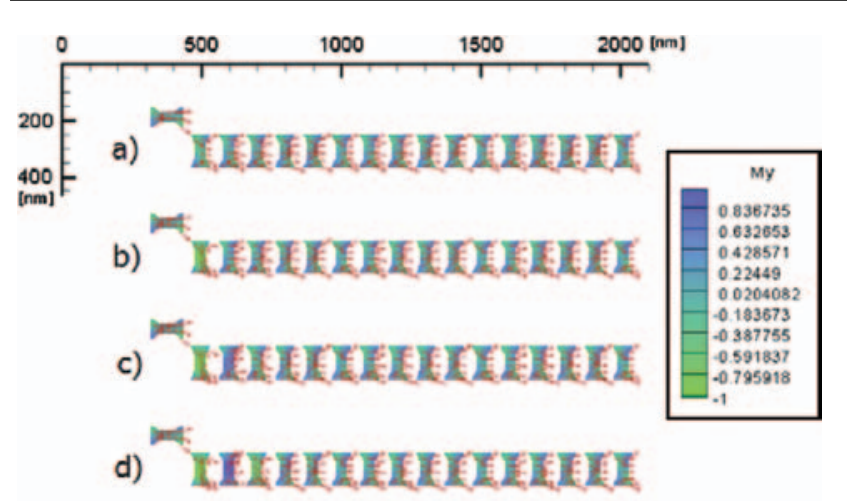

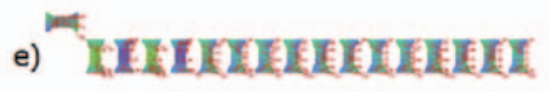

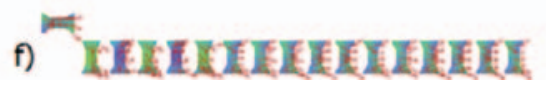

Fig. 8. Propagation of AF ordering simulated with OOMMF. The arrows point in the direction of magnetization at particular points of the nanomagnets, and the color scale is mapped to the magnetization vector in the $Y$ (vertical) direction. (a) At 1500 Oe horizontally applied clocking magnetic field, the nanomagnet wire is in the "null" state. (b) When the field is reduced to 1000 Oe, the stray-field of the input magnet starts the ordering process. From (b) to (f), the time evolution of the antiferromagnetic ordering can be followed.

emanating from the input magnet, so the whole wire settles in the state determined by the input magnet (Fig. 8). Experimental data shown in Figure 9 are in good correlation with the results of the simulations. Note that the direction of information propagation is defined by the physical placement of the horizontal magnet: switching occurs from left to right for the 1st and 3rd wires (from top to bottom) and from right to left in 2nd and 4th wires in Figure 9. Thus, the magnetization state of the input nanomagnet determines which ground-state configuration the array assumes upon removal of the clocking field.
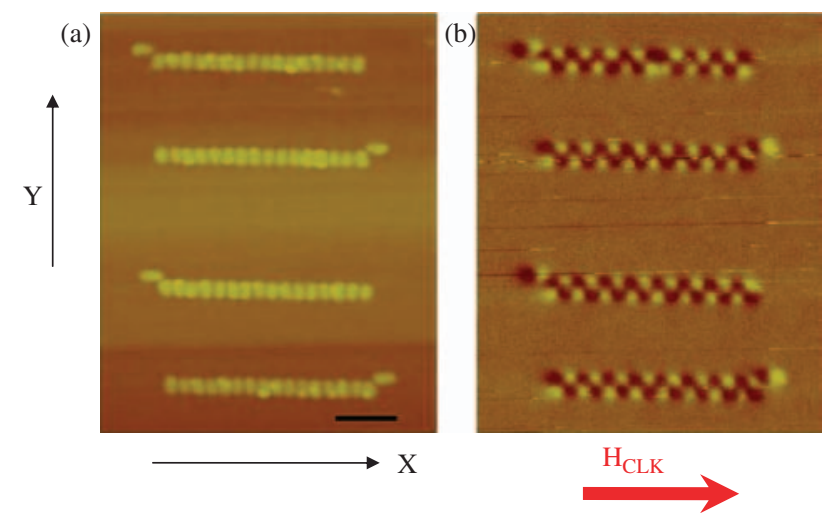

Fig. 9. Antiferromagnetic ordering in a line of nanomagnets. The ordering along the chain is controlled by an additional, horizontally-oriented elongated driver magnet. (a) AFM image of nanomagnets. (b) MFM image of the same chain shows alternating magnetization of the magnets as set by the state of the horizontal input magnets. Scaling bar length is $500 \mathrm{~nm}$. Red arrow indicates the direction of the clocking field. Note that here and below the MFM images are taken after the clocking field is removed.
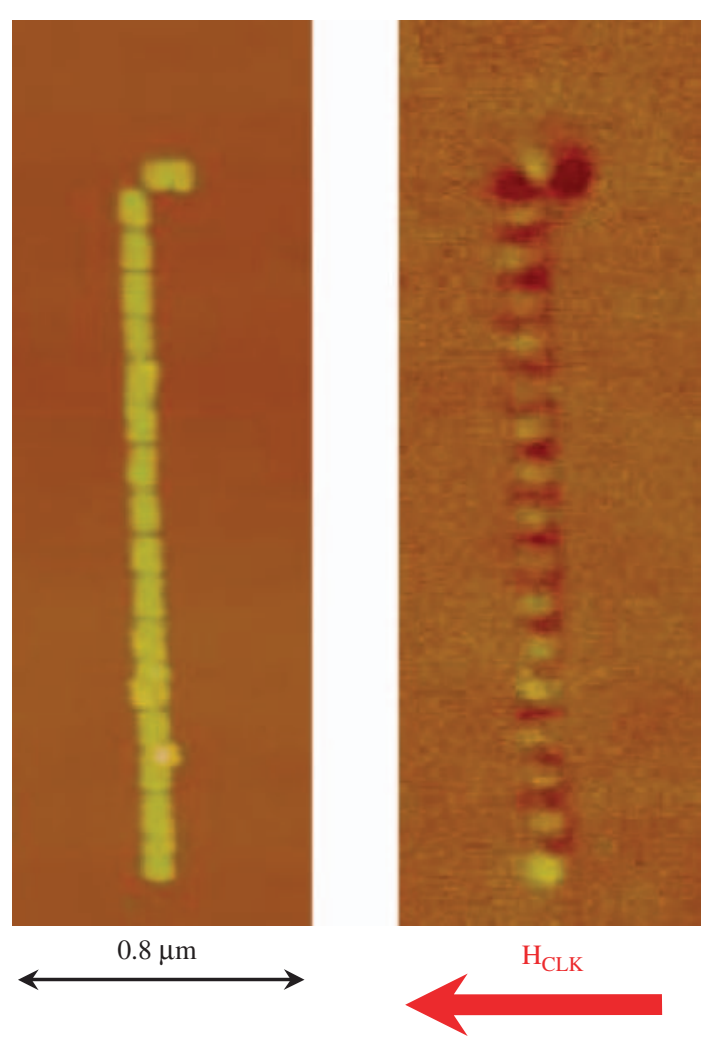

Fig. 10. AFM (left) and MFM (right) images of an F-coupled binary wire. The clocking magnetic field is oriented horizontally and indicated by the red arrow.

An error in the top binary wire in Figure 9(b) is most likely caused by the fabrication defect.

The F-coupled binary wires can similarly be operated by an external clock field while they transfer over the binary information without inversion. Figure 10 demonstrates ordering in a vertical line as a result of a horizontally applied clocking field: ${ }^{f}$ all the magnets are aligned in vertical direction in accordance with the magnetization of the input magnet at the top of the wire.

\section{MQCA DEVICES: MAJORITY LOGIC GATE}

The majority logic gate (MLG) is the key logic element of QCA computing architecture, where a binary state of the "decision making" cell is defined by the majority of the three inputs equally coupled to it. ${ }^{12}$ A MLG for MQCA that uses pillar shaped nanomagnets as cells was studied theoretically in Ref. [21]. Figure 11 shows a physical layout, schematic representation, and a truth

\footnotetext{
${ }^{f}$ The two coupling schemes, F and AF-coupled, however, showed a difference in the performance in our experiments. For the same $30 \mathrm{~nm}$ separation between the magnets, we observed a reduced tolerance of the F-coupled wire to clocking field misalignments. If the applied external clocking field has a small component in vertical direction, it can overcome the effect of the input magnet and reverse the final state of the wire. This deficiency can be avoided for pillar-shaped nanomagnets experiencing AF-coupling only.
} 
(a)

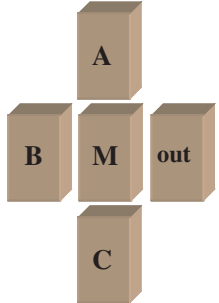

(c)

\begin{tabular}{|c|c|c|c|c|c|}
$A$ & $B$ & $C$ & $A \bar{B} C$ & $M$ & out \\
\hline 0 & 0 & 0 & 010 & 0 & 1 \\
\hline 0 & 0 & 1 & 011 & 1 & 0 \\
\hline 1 & 0 & 0 & 110 & 1 & 0 \\
\hline 1 & 0 & 1 & 111 & 1 & 0 \\
\hline 0 & 1 & 0 & 000 & 0 & 1 \\
\hline 0 & 1 & 1 & 001 & 0 & 1 \\
\hline 1 & 1 & 0 & 100 & 0 & 1 \\
\hline 1 & 1 & 1 & 101 & 1 & 0 \\
\hline
\end{tabular}

Fig. 11. Physical layout (a), schematic representation (b), and the truth table (c) for MQCA majority gate. Note that input B, and output are inverted. Decision is made based on the majority voting.

table for a MLG that uses planar magnets. The nanomagnets are arranged along two intersecting lines, where the dipole coupling of the nanomagnets produces ferromagnetic ordering along the vertical wire and antiferromagnetic ordering along the horizontal wire. Such a three-input MLG can be viewed as a programmable two-input (e.g., $A$ and C) NAND or NOR gate, depending on the state of the third gate (inverted B). Therefore, any Boolean logic function can be built by a network of majority gates.

This structure is similar to that proposed in Ref. [30] (and also studied in Ref. [31]), except that we consider the output of the gate to be in the AFC line instead of the FC line. Consider the simplest arrangement of five nanomagnets, i.e., a central nanomagnet surrounded by four others (Fig. 12(a)). Three of the neighbors (A, B, C in Fig. 12(a)) can be used as inputs driven by additional driver nanomagnets oriented in the $x$-direction, labeled $\mathrm{D}$, along the clocking field. The fourth neighbor to the right of the central magnet in Figure 12(a), is the output. The gate is constructed so that the ferromagnetic and antiferromagnetic coupling to the central, decision making nanomagnet have the same strength, ${ }^{g}$ and therefore it switches to the state to which the majority of inputs forces it. In a real world application the driver magnets will be driven by external input signals, schematically represented by magnetic field lines in Figure 12(a). One of the challenges for implementation of the real world MQCA is the need for coupling of external signals to the nanomagnets (e.g., coming from CMOS part of the circuit). Such electric-to-magnetic signal converters to be used to switch the drivers independently are not developed yet. However, to demonstrate

${ }^{g}$ Neighbor separation in the AF-coupled wire in Figure 12(b) is approximately $25 \mathrm{~nm}$; and in the F-coupled wires is about $35 \mathrm{~nm}$. This difference is intended to balance the two coupling schemes, and to set the switching field values to be similar for both wires. the majority gate function, we can make four structures with different spatial location of the driver nanomagnets (Fig. 12(b)), and then by using two opposite orientations of the clocking magnetic field all eight $(4 \times 2)$ possible input logic combinations shown in Figure 11(c) can be mimicked and tested. Simulations of the magnetic states of the majority gates after applying a horizontal clocking field show that as the clocking field decreases, switching inside the gate begins at the input magnets and then propagates to the output magnet. An example of the simulations for particular combination of input magnetic fields is shown in Figure 13. ${ }^{32}$ In the beginning (Fig. 13(a)) all three horizontal input magnets are aligned with the 5000 Oe external magnetic field, while the magnets in the majority geometry are in the null state. The switching of the magnetization starts below 2000 Oe of applied clocking field, with the magnetization of magnets "A," "B" and "C" (labels are from Fig. 12(a)) turning according to their inputs. In this case inputs " $A$ " and " $C$ " vote against the third input (inverted "B"). As a result, the decision making magnet follows the majority of the inputs. Finally, Figure 13(e) shows the relaxed magnetic state of the gate at the end of the clocking cycle.

The results of the simulations were successfully confirmed experimentally. ${ }^{33}$ Experiment also reveals that some of the tested structures have fabrication defects ${ }^{h}$ and they repeatedly show improper switching. For the structures that show correct operation of the majority-gate function, the errorless switching (with all 5 magnets in the MLG switching correctly into the state suggested by the drivers) occurs with the probability of about 0.5 . This value, however, is far greater than the probability for the MLG to randomly settle in the final state in accordance with the inputs. Indeed, the probability of all five magnets of the MLG randomly assuming the correct orientation is $0.5^{5} \approx 0.03$. One possible source of errors in the MLG operation is in the limited accuracy of the alignment of the clocking field in the experiment. Our simulations show that deviations of clocking field as small as $\pm 2^{\circ}$ degrees from horizontal axis in Figure 12(b) lead to the errors in the switching of the F-coupled wires. This deviation was hard to control in our experiment with high degree of accuracy which is likely to increase the error probability.

The inputs used in our work are set by the external clocking field and cannot be programmed independently; different combinations of the input values are realized by different physical arrangements of driver magnets. In spite of that obstacle we successfully demonstrated that the decision making nanomagnets situated in the intersection of the horizontal and vertical wires can correctly perform

\footnotetext{
${ }^{h}$ Antiferromagnetic ordering was investigated in a large set of nominally identical AF-coupled binary wires. We have found that sometimes ordering fails even in the simplest, two-magnet "wires." The identified faulty pairs performed highly repeatably, which indicates the errors to be related to fabrication variations. ${ }^{34}$
} 

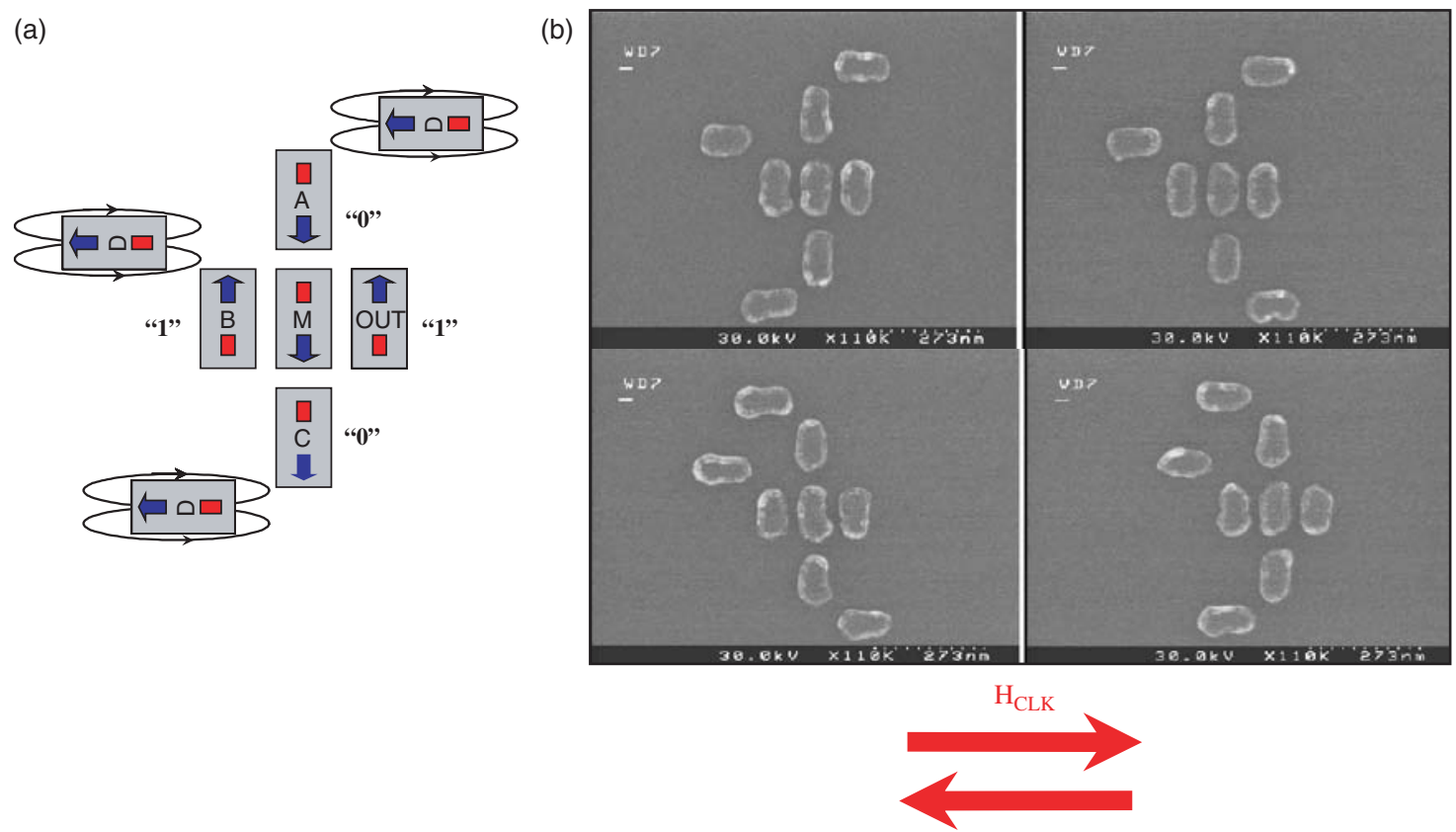

Fig. 12. (a) A sketch of MQCA majority logic gate layout. Horizontal nanomagnets labeled "D" are input drivers that must be set by local magnetic fields provided by the inputs; (b) four majority gates structures designed for testing all input combinations of the MLG. Two directions of the clocking field shown by arrows are used to magnetize input driver magnets.
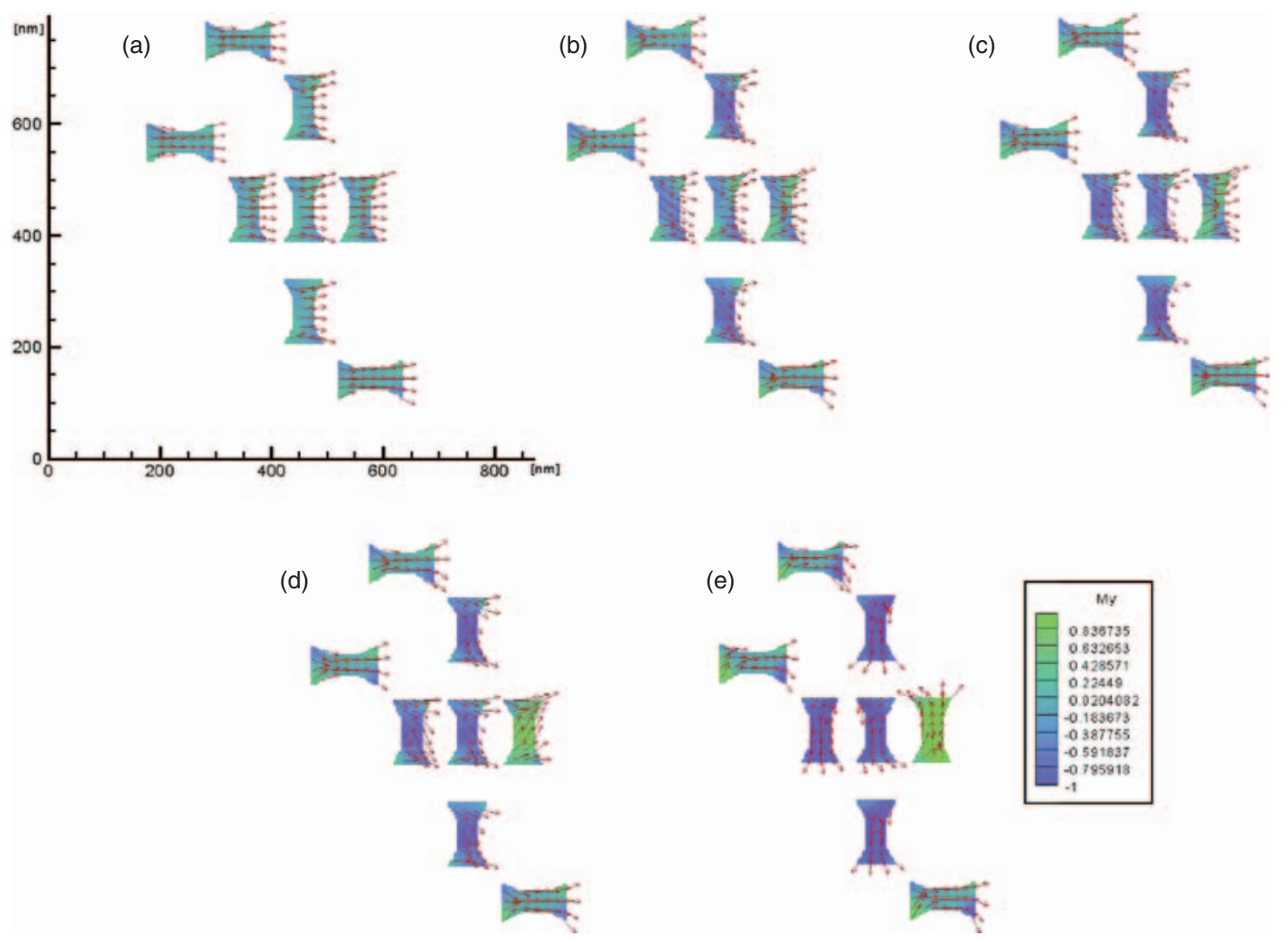

Fig. 13. Simulated dynamics of the majority operation for one combination of the inputs to MLG (000). The figures from (a) to (e) show the time-evolution of the magnetic state of the gate as the external magnetic field is ramped from 5000 Oe (a) to zero (e). 

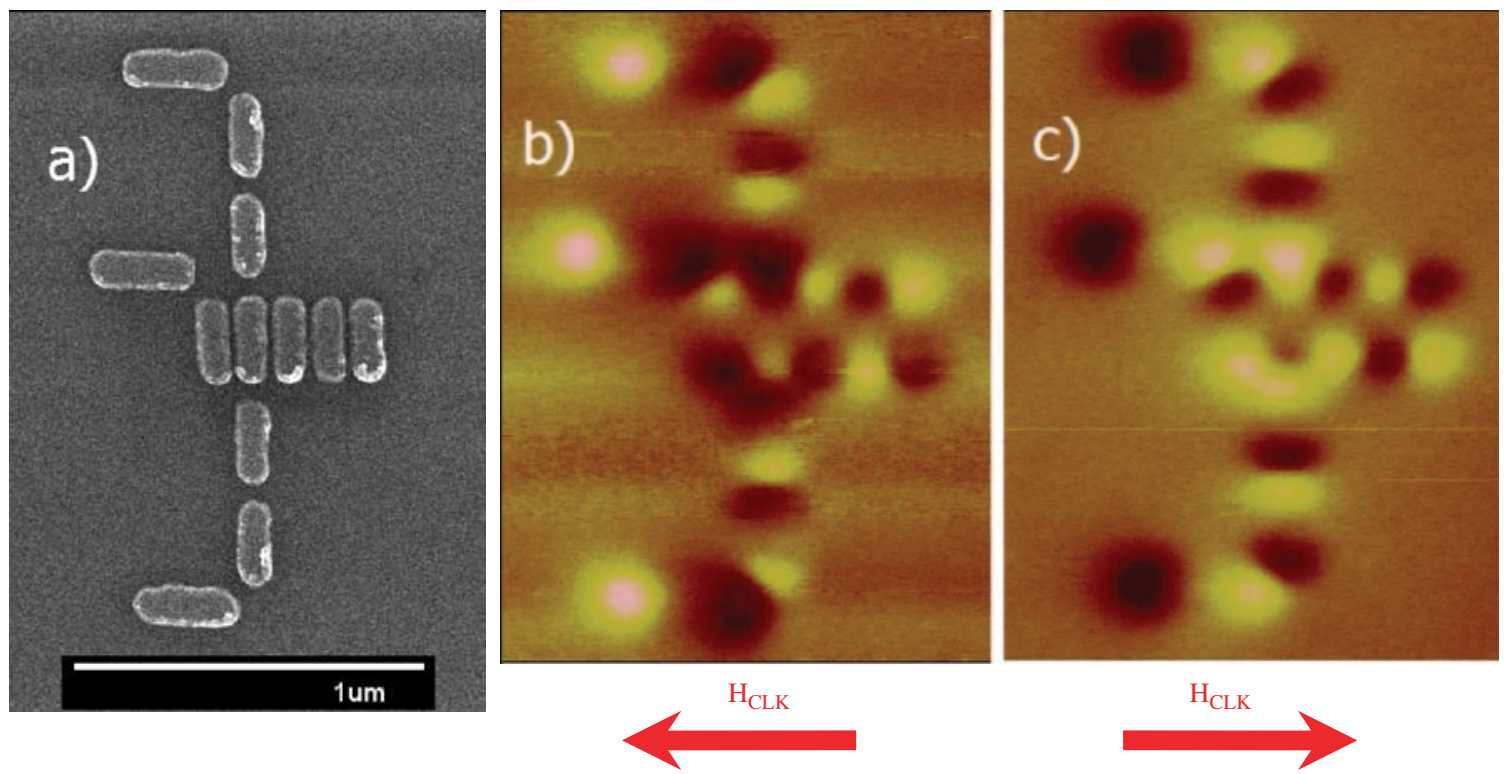

Fig. 14. An MQCA device combining a majority logic gate with binary wires. (a) Scanning electron microscope image and (b and c) magnetic force microscope images of magnetic ordering in the same gate for two orientations of horizontal clocking field, indicated by the red arrows. The $40 \mathrm{~nm}$ thick, polycrystalline permalloy nanomagnets are deposited on a silicon substrate.

the majority-logic function for all four types of tested structures.

\section{MLG-BINARY WIRE COMBINATION DEVICE}

Any logic function implemented using MQCA would require a combination of MLGs and binary wires (Fig. 6(c)). An example of a fabricated device that combines a majority gate with a binary wire of is shown in Figure 14. The micrograph (Fig. 14(a)) shows two F-coupled vertical, and one horizontal AF-coupled binary input wires reaching the decision-making magnet in the spot where these wires cross; the result of calculation is then transferred over by the AF-coupled output wire.

Figures 14(b) and (c) are MFM images taken after clocking field of 500 Oe was applied and then relaxed to zero in two opposite directions along the $X$ axis which was also setting the state of the input horizontal magnets. These images show correct alignment of all magnetic dipoles of the gates.

This example of relatively complex devices raises the question, how many nanomagnets can be switched together at the same clocking phase? We performed Monte Carlo simulations in the single-domain approximation to investigate whether the realization of larger-scale systems are feasible. ${ }^{35}$ Variations in nanomagnet shape and edgeroughness were taken into account in the distribution of the coupling fields at which switching occurs, i.e., switching fields (distribution of the demagnetization tensor elements), and thermal fluctuations were modeled by adding a stochastic field to the coupling field. We found that for our structures, the impact of switching field variations is far more important than the effect of thermal fluctuations. Strongly-coupled dots (with dot separation less than $100 \mathrm{~nm}$ ) fabricated by high-resolution lithography (with switching field variations less than $10 \%$ ) exhibit magnetic ordering over 10-20 magnets. This result agrees well with our previous experiments ${ }^{36,37}$ for samples fabricated by electron-beam lithography and lift-off. Therefore, to provide tolerance against fabrication defects, a larger-scale MQCA would require local clocking for sub-arrays that consist of only a few gates. The small number of magnets switching at the same time keeps the error level acceptably small. This concept of local clocking fields has been developed for EQCA. ${ }^{38}$ The most suitable architecture for adiabatically-clocked MQCA devices appears to be a pipelined structure. Because of the sequential arrangement of logic gates, there will inevitably be pipeline latency, however new data can be fed into the pipeline at each clock cycle. Clocking zones can be defined by locally applied clocking fields. Pipelined architectures are generally desirable due to their highly parallelized computing environment. A realistic pipelined clocking scheme in which the current in a yoked wire creates a sufficiently high magnetic field to cause all of the magnets in one stage of the pipe into the null state has been recently suggested. ${ }^{27}$ Proper clock phasing causes the data to pass from stage to stage.

\section{DEVELOPMENT OF THE INPUT AND READOUT FOR MQCA}

A vital issue for any all-magnetic logic device is its ability to interact with outside electronics. Therefore, the development of a reliable MQCA interface converting electrical signals to magnetic fields, and vice versa, remains crucial. 
Integration of MQCA elements with electronic circuitry may be possible in a manner similar to magnetic random access memories (MRAM). ${ }^{39}$ Furthermore, integration of MQCA arrays into MRAM cells is also feasible, thus allowing the possibility of "intelligent memory" where the magnetic layer of an MRAM cell could not only store a single bit of information, but could also be capable of performing some basic logical processing. This may provide an opportunity to increase the functionality and integration density of an MRAM device.

Figure 15(a) shows a sketch of majority gate MQCA with attached inputs and outputs. The input-interfacing device, in principle, could be built using current carrying wires placed perpendicular to the input nanomagnets, in which case locally generated magnetic fields can switch the magnetization of the input nanomagnets. The outputinterfacing device must non-invasively read the state of the MQCA logic gate and convert it to a measurable electrical signal. There are several possible sensing mechanisms that could be utilized as readouts for MQCA. One promising readout scheme is based upon the resistance change in a magnetic wire caused by the trapping of a domain wall by the output nanomagnet in the MQCA circuit (Fig. 15(b)). Such trapping of a domain wall leads to a measurable $(\sim 1 \%)$ change in the resistance of the wire: for a domain wall trapped between the potential leads, the resistance decreases by a fraction proportional to the volume of the domain wall. It is also possible to employ a nanoscale Hall sensor under the nanomagnet at the edge of MQCA array ${ }^{40,41}$ (Fig. 16(a)) and measure a change in the resistance for the two remanent states of the magnet (Fig. 16(b)). Another potential solution would be to use a magnetoresistive sensor similar to that used in MRAM. It would be difficult, however, to have an on-chip sensor that would not be affected by the clocking field and at the same time not influence the state of the MQCA. One

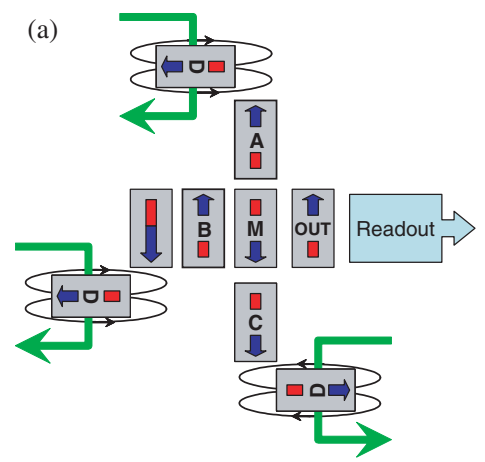

(b)

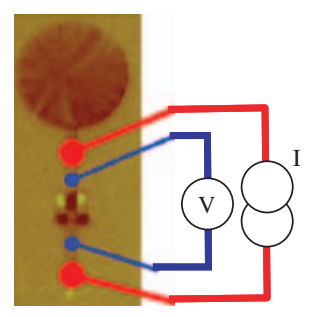

Fig. 15. Interfacing MQCA. (a) Schematic representation of MQCA majority gate with input and output interface. Green lines represent current carrying wires generating local magnetic fields. (b) A possible implementation of a readout device. The MFM image shows a domain wall trapped in a magnetic wire between two nearby nanomagnets. The structure is made of $40 \mathrm{~nm}$ thick permalloy, and the nanomagnets are separated from the wire by $60 \mathrm{~nm}$. Four probe circuit can be used to measure the resistance change due to domain wall trapping. (a)
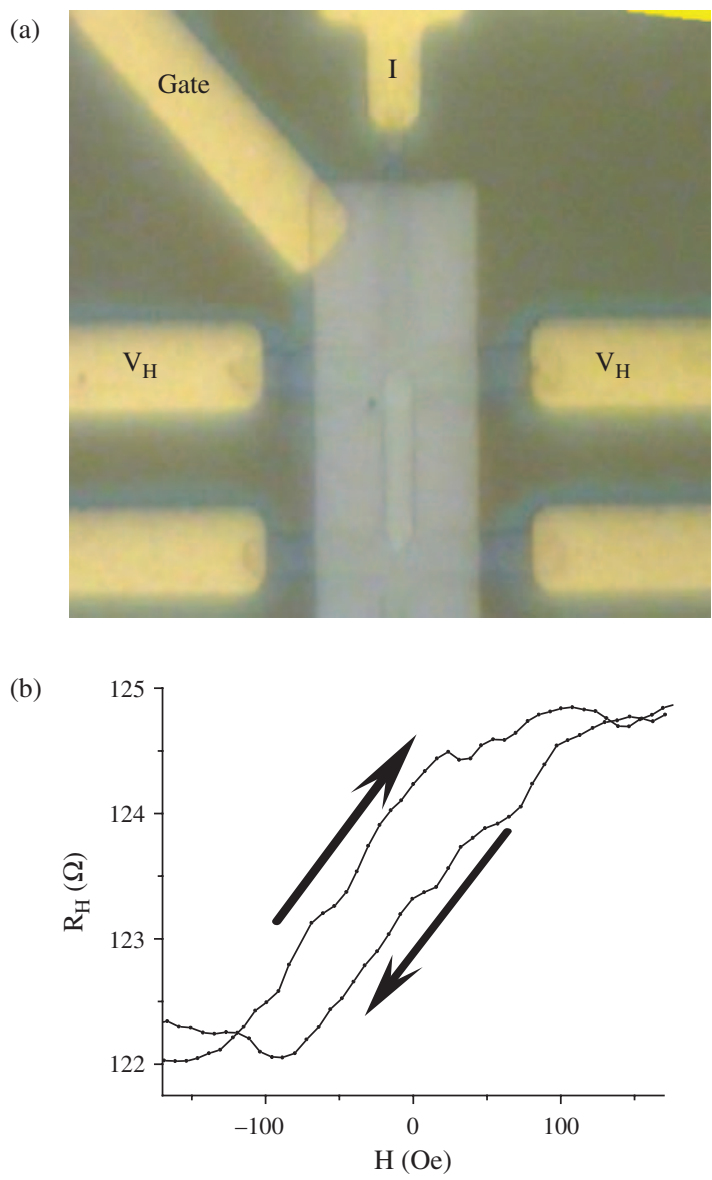

Fig. 16. Hybrid Si MOS-Hall effect device. (a) Optical micrograph of the device showing a magnet $\left(2 \times 19 \mu \mathrm{m}^{2}, 150 \mathrm{~nm}\right.$ thick $)$ on top of the gate; Hall electrodes are labeled as $V_{\mathrm{H}}$, one current lead $(I)$ and the gate wire are shown. (b) Hall resistance measurement in magnetic field along the easy axis of the magnet reveals resistance change of about $1 \%$ for the two remanent states of the magnet for gate voltage of $4 \mathrm{~V}$. Arrows indicate the directions of the magnetic field ramps.

possible way to avoid this problem would be to employ some MEM-based positioning system (similar to one used in hard drives or cantilever-like mechanism) to bring the GMR sensor close to the output magnets for the readout procedure only. We are currently investigating these options.

\section{ALTERNATIVE TECHNIQUES FOR MQCA FABRICATION}

A very important issue for making MQCA viable is to eliminate fabrication errors that are presently limiting the size of logic gates as well as the number of nanomagnets acting together. The appropriate choice of fabrication technique must provide extremely dense arrays of precisely placed single-domain nanomagnets of uniform size and shape, and at the same time should allow the custom design of various MQCA network layouts. The latter suggests the application of certain lithographic 
techniques on the scale of the logic gates, while the requirement of highly uniform nanomagnets demands a true nanofabrication technique. Fortunately, our quest for the appropriate MQCA technology may be able to follow the current developments in the magnetic recording hard disk drive (HDD) industry. To further increase the bit density of HDDs, continuous thin-film magnetic media is being replaced by a pre-patterned media ${ }^{42}$ where the bits are stored as the magnetization of single-domain nanoelements, just as in the case of MQCA. Further similarities are that the nanomagnets are placed in regular arrays, and have strict requirement for uniformity. In patterned magnetic media, the HDD head addresses the nanomagnets individually, which improves the signal to noise ratio of the read-write process as compared to the thin-film media, and thus enables the signal, i.e., the magnetization of the nanomagnets to be reduced. This leads to reduced volume, which ensures disk storage density over $40 \mathrm{Gbits} / \mathrm{in}^{2}$, possibly reaching to Tbits/in ${ }^{2}{ }^{43}$ Several different fabrication techniques for producing patterned magnetic media that are currently under development are discussed in detail in recent publications. ${ }^{43-45}$ In addition to the conventional fabrication techniques, active search in guided self-assembly of magnetic nanoparticles from solutions ${ }^{46}$ is under way.

\section{SUMMARY}

In summary, we demonstrated the operation of the key elements for magnetic QCA computational architecture: ferromagnetically and antiferromagnetically coupled binary wires, majority logic gates, and the combination of the two. We proved that logic functions can be realized in properly-structured arrays of physically-coupled nanomagnets where the switching is controlled by clocking magnetic field. The technology for fabricating such nanometer scale magnets is currently under development by the hard disk drive industry. While that latter work focuses entirely on data-storage applications, where physical coupling between individual bits is undesirable, our work points out the possibility of realizing all-magnetic logic functionality in such systems, and indicates the potential of all-magnetic information processing systems that incorporate both memory and logic.

Acknowledgments: The authors wish to thank G. L. Snider for reading the manuscript and numerous valuable suggestions. Our work is supported in part by grants from the Office of Naval Research, the W. M. Keck Foundation, and the National Science Foundation.

\section{References and Notes}

1. D. H. Looney, J. Appl. Phys. 30 (1959).

2. D. R. Bennion, H. D. Crane, and D. Nitzan, Digital Magnetic Logic, McGraw-Hill, New York (1969).
3. S. Scrupski, 40 years ago: Magnetic computer uses multiaperture cores, Electronic Design (2001).

4. R. J. Spain, J. Appl. Phys. 37 (1966).

5. R. J. Spain and H. I. Jauvtis, J. Appl. Phys. 37 (1966).

6. R. J. Spain and H. I. Jauvtis, J. Appl. Phys. 38 (1967).

7. S. Y. Lee and H. Chang, IEEE Transactions on Magnetics MAG-10, 1059 (1974).

8. A. Ney, C. Pampuch, R. Koch, and K. H. Ploog, Nature 425 (2003).

9. D. A. Allwood, G. Xiong, C. C. Faulkner, D. Atkinson, D. Petit, and R. P. Cowburn, Science 309, 1688 (2005).

10. G. Csaba, P. Porod, and A. I. Csurgay, International Journal of Circuit Theory and Applications 31, 67 (2003).

11. J. De Boeck, W. Van Roy, J. Das, V. Motsnyi, Z. Liu, L. Lagae, H. Boeve, K. Dessein, and G. Borghs, Semicond. Sci. Technol. 17, 342 (2002).

12. C. S. Lent, P. D. Tougaw, W. Porod, and G. H. Bernstein, Nanotechnology 4, 49 (1993)

13. A. O. Orlov, I. Amlani, G. H. Bernstein, C. S. Lent, and G. L. Snider, Science 277, 928 (1997).

14. I. Amlani, A. O. Orlov, G. Toth, G. H. Bernstein, C. S. Lent, and G. L. Snider, Science 284, 289 (1999).

15. A. O. Orlov, R. Kummamuru, R. Ramasubramaniam, C. S. Lent, G. H. Bernstein, and G. L. Snider, Surf. Sci. 532-535, 1193 (2003).

16. K. K. Yadavalli, A. O. Orlov, J. P. Timler, C. S. Lent, and G. L. Snider, Nanotechnology 18, 4 (2007).

17. A. O. Orlov, R. K. Kummamuru, J. Timler, C. S. Lent, G. L. Snider, and G. H. Bernstein, Experimental studies of quantum-dot cellular automata devices, Mesoscopic Tunneling Devices, edited by H. Nakashima (2004), pp. 125-160.

18. C. S. Lent and B. Isaksen, IEEE Transactions on Electron Devices 50, 1890 (2003).

19. C. S. Lent, B. Isaksen, and M. Leiberman, J. Am. Chem. Soc. 125, 1056 (2003).

20. G. Csaba, A. Imre, G. H. Bernstein, W. Porod, and V. Metlushko, IEEE Trans. Nanotechnol. 209 (2002).

21. G. Csaba and W. Porod, Journal of Computational Electronics 1, 87 (2002).

22. R. P. Cowburn and M. E. Welland, Science 287 (2000).

23. U. F. Gianola, J. Appl. Phys. 32 (1961).

24. M. P. Anantram and V. P. Roychowdhury, J. Appl. Phys. 85, 1622 (1999).

25. G. Csaba, P. Luigi, A. Csurgay, and W. Porod, Journal of Computational Electronics 4, 105 (2005).

26. T. Gerrits, H. A. M. Van den Berg, J. Hohlfeld, L. Bar, and T. Rasing, Nature 418, 509 (2002).

27. M. T. Niemier, X. S. Hu, M. Alam, G. Bernstein, W. Porod, M. Putney, and J. DeAngelis, Clocking structures and power analysis for nanomagnet-based logic devices (2007).

28. M. J. Donahue and D. G. Porter, OOMMF User's Guide, Version 1.0 ed: Interagency Report NISTIR 6376 (2000).

29. A. Imre, G. Csaba, J. Lili, A. Orlov, G. H. Bernstein, V. Metlushko, and W. Porod, Field-Coupled Nanomagnets for Logic Applications, Sevilla, Spain (2005), pp. 162-70.

30. M. C. B. Parish and M. Forshaw, Appl. Phys. Lett. 83, 2046 (2003).

31. S. A. Haque, M. Yamamoto, R. Nakatani, and Y. Endo, Binary Logic Gates by Ferromagnetic Nanodots, Taipei, Taiwan (2004), pp. 380-4.

32. A. Imre, Ph.D. Thesis, University of Notre Dame (2005).

33. A. Imre, G. Csaba, L. Ji, A. Orlov, G. H. Bernstein, and W. Porod, Science 311, 205 (2006).

34. G. H. Bernstein, A. Imre, V. Metlushko, A. Orlov, L. Zhou, L. Ji, G. Csaba, and W. Porod, Microelectron. J. 36, 619 (2005).

35. G. Csaba, Ph.D. Thesis, University of Notre Dame (2003).

36. A. Imre, G. Csaba, G. H. Bernstein, W. Porod, and V. Metlushko, Investigation of Shape-Dependent Switching of Coupled Nanomagnets, Maui, HI, USA (2003), pp. 513-18. 
37. A. Imre, G. Csaba, G. H. Bernstein, W. Porod, and V. Metlushko, Investigation of Antiferromagnetic Ordering Along Chains of Coupled Nanomagnets, San Francisco, CA, USA (2003), pp. 20-3.

38. J. Timler and C. S. Lent, J. Appl. Phys. 91, 823 (2002).

39. J. M. Slaughter, R. W. Dave, M. DeHerrera, M. Durlam, B. N. Engel, L. Janesky, N. D. Rizzo, and S. Tehrani, Fundamentals of MRAM Technology, Washington, DC, USA (2002), pp. 19-25.

40. M. Johnson, B. R. Bennett, M. J. Yang, M. M. Miller, and B. V. Shanabrook, Appl. Phys. Lett. 71, 974 (1997).

41. P. Das Kanungo, A. Imre, B. Wu, A. Orlov, G. Snider, W. Porod, and N. P. Carter, Microelectron. J. 36, 294 (2005).
42. D. A. Thompson and J. S. Best, IBM Journal of Research and Development 44,311 (2000).

43. B. D. Terris and T. Thomson, J. Phys. D: (Appl. Phys.) 38, 199 (2005).

44. J. I. Martin, J. Nogues, L. Kai, J. L. Vicent, and I. K. Schuller, J. Magn. Magn. Mater. 256, 449 (2003).

45. C. A. Ross, Annu. Rev. Mater. Sci. 31, 203 (2001).

46. R. Lirong, G. Lin, M. Wark, and Y. Hou, Appl. Phys. Lett. 87, 212503 (2005).

47. A. Orlov, A. Imre, L. Ji, G. Csaba, G. Bernstein, and W. Porod, ECS Transactions 25, 317 (2007).

Received: 17 December 2007. Revised/Accepted: 20 December 2007. 